\title{
Acknowledgment of Reviewers
}

The Editor gratefully acknowledges the assistance of the following persons who reviewed manuscripts submitted to the Journal of Diversity in Higher Education within the past year.

Nancy Acevedo-Gil

Fenaba Addo

Matthew Aguirre

Rachel Aho

Cynthia Maribel Alcantar

Franklin Allaire

Katherine Aquino

Raquel Arouca

Ann Austin

Sofia Bahena

Aldo Barrita

Michael Bastedo

Denise A. Battles

Aryn Baxter

Kevin J. Bazner

Jessica Belue Buckley

Mimi Benjamin

Jessica Bennett

Bianca Bernstein

Genia Bettencourt

Jennifer M. Blaney

Fred A. Bonner

Nicholas Bowman

Allison BrckaLorenz

Roshaunda Breeden

Kaleb Briscoe

Derrick R. Brooms

Blakely Brown

Kim Bullington

Rebecca Burch

David A. Caicedo

Cierra Campbell-Bridges*

Vincent D. Carales

Ashley Carpenter

Collette Chapman-Hilliard

Cheryl Ching

Carmen R. Cid

Monique M. Crandal

Delando Crooks*

Marcela G. Cuellar

George Cunningham

Bradley D. Custer

Alex Czopp

Amalia Dache

Kun Dai

Kirsten Davis

Tiffany Davis

Mary Ellen Dello Stritto
Jesse Michael Denton

James DeVita

Erich Dietrich

Antonio Duran

Pamela Eddy

Rebecca Evan

Nancy J. Evans

Eric Felix

Frank Fernandez

Kristie Ford

Kevin Fosnacht

Zak Foste

Antoinette Freitas

Nichole Garcia

Claudia Garcia-Louis

Stacey D. Garrett

Jason C. Garvey

Regina Garza Mitchell

Tanya Gaxiola Serrano

Kari George

Alberta Marie Gloria

Allison Godwin

Kimberly Griffin

Tonia Guida

Danielle Guzman-Orth

Candace N. Hall

Suejung Han

Yi Hao

Casandra Harper

Nicholas Hartlep

Chayla Haynes

Troy Heffernan

T. T. Heng

Paul R. Hernandez

Susana H. Hernandez

Catherine Horn

Xiaodan $\mathrm{Hu}$

Sarah Hurtado

Terry Ishitani

Grant R. Jackson

Michelle Jacob

Dimpal Jain

Chaddrick Donavan

James-Gallaway

Christopher Jett

Jennifer Michelle Johnson

Tamara A. Johnson
Veronica Jones

Darold Joseph

Fariba Karimi

Tehmina Khwaja

Dongbin Kim

Stephanie Kim

Young Hwa Kim

Ezekiel Kimball

Joseph Kitchen

David Knight

Katie Koo

Masha Krsmanovic

V. Leilani Kupo

Lisa Lambert Snodgrass

Sandra Laursen

María C. Ledesma

Elizabeth Lee

Hang-Shim Lee

Matthew Lee

Raul Leon

Luis Antonio Leyva

Jodi Linley

Tao Liu

Leslie Locke

Jameson David Lopez

Adam Love

Jacqueline Mac

Victoria K. Malaney

Brown

Ketevan Mamiseishvili

Dina Maramba

Susan Marine

Sarah Marshall

Eligio Martinez

Kelly Maxwell

Ann Maydosz

Stephanie McClure

Keon M. McGuire

Pilar Mendoza

Monica Miles

Kenneth L. Miller

Robin Minthorn

Joya Misra

Joshua James Mitchell

Vanessa Monterosa

Amanda R. Morales

Karen Moran Jackson

Jeana E. Morrison
Tierra Moss*

Samuel Museus

Brett Ranon Nachman

Mitsu Narui

Federick Ngo

Arlette J. Ngoubene-Atioky

Elizabeth Niehaus

Evangela Q. Oates

KerryAnn O'Meara

Ifeyinwa Onyenekwu

Cecilia Orphan

Robert T. Palmer

Eugene T. Parker

Jenelle S. Pitt

Luis Ponjuán

OiYan Poon

Rachele L. Pope

Stephen Porter

Julie Renee Posselt

Jonathan T. Pryor

Wei Qi

Delma Ramos

Kristen A. Renn

Sharon Robinson Kurpius

Sarah L. Rodriguez

Sophia Rodriguez

Yedalis Ruiz

Cinthya Salazar

Cristobal Salinas Jr

Margaret Sallee

Berenice Sanchez

Vanessa Sansone

Stephen Santa-Ramirez

Linda Sax

Megan Segoshi

John Singer

Christopher Slaten

Kamia Slaughter*

Brittany Smotherson*

Patricia Somers

Lisa Beth Spanierman

Elizabeth Sparks

Kat Stephen*

Saran Stewart

Terah J. Stewart

Terrell Lamont Strayhorn 
Thandi Sule

Chang Su-Russell

Susan Swayze

\section{Jason Taylor}

Leonard Taylor

M. Yvonne Taylor*

Aimee LaPointe Terosky
Tenisha Tevis

Lisette E. Torres-Gerald

Susan Torres-Harding

Teniell L. Trolian

Deborah Trytten

Marina Tulin

Jim Vander Putten
Marissa Vasquez

Tiffany Viggiano

Cynthia Villarreal*

Louise Michelle Vital

Bevo Wahono

Thomas E. Walker

Xueli Wang
Trevor Warburton

John C. Weidman

Rachelle Winkle-Wagner

Lisa Wolf-Wendel

Jacqueline $\mathrm{Yi}^{*}$

Varaxy Yi

Desiree Zerquera

* Denotes a predoctoral co-reviewer. 